\title{
A COMPLEMENTARY TRIANGLE INEQUALITY IN HILBERT AND BANACH SPACES
}

\author{
J. B. DIAZ AND F. T. METCALF ${ }^{1}$
}

1. Introduction. In a recent paper [1], Wilf has given an extension of the arithmetic-geometric mean inequality to the case of complex numbers. His result may be stated as follows:

Theorem (WiLf). Suppose the complex numbers $z_{1}, \cdots, z_{n}$, whenever $z_{i} \neq 0$, satisfy

$$
\left|\arg z_{i}\right| \leqq \psi \leqq \frac{\pi}{2}, \quad i=1,2, \cdots, n .
$$

\section{Then}

$$
(\cos \psi)\left|z_{1} z_{2} \cdots z_{n}\right|^{1 / n} \leqq 1 / n\left|z_{1}+z_{2}+\cdots+z_{n}\right|,
$$

where equality holds if and only if either:

$$
\begin{aligned}
& \psi \neq 0, n \text { even, and (after rearrangement, if necessary) } \\
& z_{1}=\cdots=z_{n / 2}=\bar{z}_{(n / 2)+1}=\cdots=\bar{z}_{n}=R \cdot e^{i \psi}
\end{aligned}
$$

or else

$$
\psi=0 \text { and } z_{1}=\cdots=z_{n} .
$$

In the course of his proof of inequality (2), Wilf derives as an intermediate auxiliary inequality the following:

$$
(\cos \psi)\left(\left|z_{1}\right|+\cdots+\left|z_{n}\right|\right) \leqq\left|z_{1}+\cdots+z_{n}\right| .
$$

Since inequality (2) follows readily from (3) by an application of the arithmetic-geometric mean inequality for real numbers, it is clear that inequality (3) plays the more fundamental rôle. In fact, inequality (3) may be interpreted as a "complementary" triangle inequality, i.e., an inequality which "runs the other way" from the usual triangle inequality. The complementary character of (3), relative to the usual triangle inequality may be described as follows. The usual triangle inequality states that, for any complex $z_{1}, \cdots, z_{n}$, one has

$$
\begin{aligned}
0 \cdot\left(\left|z_{1}\right|+\cdots+\left|z_{n}\right|\right) & \leqq\left|z_{1}+\cdots+z_{n}\right| \\
& \leqq 1 \cdot\left(\left|z_{1}\right|+\cdots+\left|z_{n}\right|\right) .
\end{aligned}
$$

Received by the editors February 15, 1965.

1 The research of the authors was supported in part by the Air Force Office of Scientific Research-Grant AFOSR 400-64. 
On the other hand, (3) states that, for suitably restricted $z_{1}, \cdots, z_{n}$ (i.e., such that $\left|\arg z_{i}\right| \leqq \psi \leqq \pi / 2$ for $i=1, \cdots, n$ ), the trivial constant zero on the left of (4) can be replaced by $\cos \psi$ (notice, however, that even in these restricted circumstances the constant one on the right of (4) cannot be replaced by a smaller constant).

Inequalities which are "complementary" to the Cauchy inequality for finite sums, to the Buniakowsky-Schwarz inequality for integrals, etc., are to be found in the literature, see [2] and [3].

Hypothesis (1) may be interpreted geometrically as requiring that the complex numbers in question lie within a cone of aperture $2 \psi \leqq \pi$, with vertex at the origin, and which is symmetric about the real axis. This last assumption is, however, not essential (one could assume that there is a real number $\theta$ such that $\left|\arg z_{i}-\theta\right| \leqq \psi \leqq \pi / 2$, which would merely mean a rotation of the original cone through an angle $\theta$ ).

The main purpose of the present note is to extend the complementary triangle inequality (3), first to a Hilbert space, and then to a Banach space. Here one can again interpret geometrically the hypothesis as requiring certain vectors to lie within a "cone."

2. Complementary triangle inequality in Hilbert space. Let $H$ be a Hilbert space, with real or complex scalars. Then one has the following analogue of (3):

TheOREM 1. Let a be a unit vector in $H$. Suppose the vectors $x_{1}, \cdots$, $x_{n}$, whenever $x_{i} \neq 0$, satisfy

$$
0 \leqq r \leqq \frac{\operatorname{Re}\left(x_{i}, a\right)}{\left\|x_{i}\right\|}, \quad i=1, \cdots, n .
$$

Then

$$
r\left(\left\|x_{1}\right\|+\cdots+\left\|x_{n}\right\|\right) \leqq\left\|x_{1}+\cdots+x_{n}\right\|,
$$

where equality holds if and only if

$$
x_{1}+\cdots+x_{n}=r\left(\left\|x_{1}\right\|+\cdots+\left\|x_{n}\right\|\right) a .
$$

Proof. In view of the Schwarz inequality, applied to the vector $x_{1}+\cdots+x_{n}$ and the unit vector $a$,

$$
\begin{aligned}
\left\|x_{1}+\cdots+x_{n}\right\| & \geqq\left|\left(x_{1}+\cdots+x_{n}, a\right)\right| \\
& \geqq\left|\operatorname{Re}\left(x_{1}+\cdots+x_{n}, a\right)\right| \\
& =\left|\operatorname{Re}\left(x_{1}, a\right)+\cdots+\operatorname{Re}\left(x_{n}, a\right)\right| .
\end{aligned}
$$

Now, by hypothesis (5), 


$$
\begin{aligned}
\left|\operatorname{Re}\left(x_{1}, a\right)+\cdots+\operatorname{Re}\left(x_{n}, a\right)\right| & =\operatorname{Re}\left(x_{1}, a\right)+\cdots+\operatorname{Re}\left(x_{n}, a\right) \\
& \geqq r\left(\left\|x_{1}\right\|+\cdots+\left\|x_{n}\right\|\right),
\end{aligned}
$$

which yields (6).

Now for the equality condition in (6). If (7) holds, then it is clear that equality holds in (6). Next, suppose the equality sign holds in (6). Then it holds at every intermediate inequality in the argument just given. That is to say, one has

$$
\begin{aligned}
& \text { (a) } x_{1}+\cdots+x_{n}=\left(x_{1}+\cdots+x_{n}, a\right) a \\
& \text { (b) } \operatorname{Im}\left(x_{1}+\cdots+x_{n}, a\right)=0
\end{aligned}
$$

and

$$
\text { (c) } \operatorname{Re}\left(x_{i}, a\right)=r\left\|x_{i}\right\|, \quad \text { for } i=1, \cdots, n \text {. }
$$

Hence,

$$
\begin{aligned}
\left(x_{1}+\cdots+x_{n}, a\right) & =\operatorname{Re}\left(x_{1}+\cdots+x_{n}, a\right) \\
& =\operatorname{Re}\left(x_{1}, a\right)+\cdots+\operatorname{Re}\left(x_{n}, a\right) \\
& =r\left(\left\|x_{1}\right\|+\cdots+\left\|x_{n}\right\|\right),
\end{aligned}
$$

which, together with (a), gives (7).

REMARK 1. Hypothesis (5) may be rewritten in a form which resembles hypothesis (1) of Wilf's theorem. One has merely to put $r=\cos \psi$, with $0 \leqq \psi \leqq \pi / 2$, to obtain from (5) the equivalent inequality

$$
\operatorname{Cos}^{-1}\left(\frac{\operatorname{Re}\left(x_{i}, a\right)}{\left\|x_{i}\right\|}\right) \leqq \psi \leqq \frac{\pi}{2} .
$$

Notice also that, in order to avoid distinguishing between zero and nonzero $x_{i}$, it may be better to rewrite hypothesis (5) as follows:

$$
0 \leqq r x_{i} \| \leqq \operatorname{Re}\left(x_{i}, a\right), \quad i=1, \cdots, n .
$$

In the alternative form $\left(5^{\prime}\right)$, the hypothesis already looks a lot like the conclusion of the theorem.

COROLlaRy 1. Under the hypotheses of Theorem 1, one has

$$
r\left(\left\|x_{1}\right\| \cdots\left\|x_{n}\right\|\right)^{1 / n} \leqq \frac{1}{n}\left\|x_{1}+\cdots+x_{n}\right\|
$$




$$
r\left(\frac{\left\|x_{1}\right\|^{p}+\cdots+\left\|x_{n}\right\|^{p}}{n}\right)^{1 / p} \leqq \frac{1}{n}\left\|x_{1}+\cdots+x_{n}\right\|,
$$

where $p<1$ and $p \neq 0$. Equality holds in (8) (or in (9)) if and only if

$$
x_{1}+\cdots+x_{n}=r\left(\left\|x_{1}\right\|+\cdots+\left\|x_{n}\right\|\right) a
$$

and

$$
\left\|x_{1}\right\|=\cdots=\left\|x_{n}\right\| .
$$

Proof. From Hardy, Littlewood, and Pólya [4, p. 26],

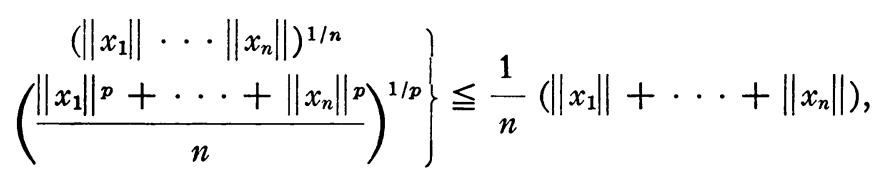

with equality if and only if $\left\|x_{1}\right\|=\cdots=\left\|x_{n}\right\|$. This, together with Theorem 1, gives the desired result.

It should be noted that (8) can be thought of as taking $p=0$ in (9). Also, the apparently excluded case of $p=1$ is just Theorem 1 itself, where the condition $\left\|x_{1}\right\|=\cdots=\left\|x_{n}\right\|$ is not a part of the equality condition.

REMARK 2. In order to see that Wilf's theorem is a special case of Corollary 1 , one need only take $H$ to be the complex numbers with the usual scalar product, $\left(z_{1}, z_{2}\right)=z_{1} \bar{z}_{2}$; the norm being the usual absolute value, $\|z\|=|z|$. Putting $a=1$ and $r=\cos \psi$, with $0 \leqq \psi \leqq \pi / 2$, in (5) gives hypothesis (1), since then $\operatorname{Re}\left(x_{i}, a\right)$ is just $\operatorname{Re} x_{i}$. The equality condition in Corollary 1 implies that equality holds in Wilf's theorem if and only if

$$
x_{1}+\cdots+x_{n}=r\left(\left|x_{1}\right|+\cdots+\left|x_{n}\right|\right)
$$

and

$$
\left|x_{1}\right|=\cdots=\left|x_{n}\right| \quad(=\lambda, \text { say }) .
$$

Transposing, and taking the real part of (10) gives

$$
\left(\operatorname{Re} x_{1}-r\left|x_{1}\right|\right)+\cdots+\left(\operatorname{Re} x_{n}-r\left|x_{n}\right|\right)=0
$$

which means that

$$
\operatorname{Re} x_{k}=r\left|x_{k}\right|, \quad \text { for } k=1, \cdots, n .
$$

Thus,

$$
x_{k}=\left|x_{k}\right|\left(r \pm i\left(1-r^{2}\right)^{1 / 2}\right)=\lambda\left(r \pm i\left(1-r^{2}\right)^{1 / 2}\right)
$$


where the choice of sign depends on $k$. Let $j$ be the number of $x_{k}$ 's for which the positive square root holds. Then

$$
x_{1}+\cdots+x_{n}=\lambda\left[n r+i(j-(n-j))\left(1-r^{2}\right)^{1 / 2}\right] .
$$

Since, from (10), the imaginary part of $x_{1}+\cdots+x_{n}$ is zero, the equality condition of Wilf's theorem follows.

Corollary 2. Let the "weights" $q_{1}, \cdots, q_{n}$ be real, positive, and such that $q_{1}+\cdots+q_{n}=1$. Under the hypotheses of Theorem 1 one has

$$
\left.r\left\|x_{1}\right\| q_{1} \cdots\left\|x_{n}\right\|\right|^{a_{n}} \leqq\left\|q_{1} x_{1}+\cdots+q_{n} x_{n}\right\|
$$

and

$$
r\left(q_{1}\left\|x_{1}\right\|^{p}+\cdots+q_{n}\left\|x_{n}\right\|^{p}\right)^{1 / p} \leqq\left\|q_{1} x_{1}+\cdots+q_{n} x_{n}\right\|,
$$

where $p<1$ and $p \neq 0$. Equality holds in (12) (or in (13)) if and only if

$$
q_{1} x_{1}+\cdots+q_{n} x_{n}=r\left(q_{1}\left\|x_{1}\right\|+\cdots+q_{n}\left\|x_{n}\right\|\right) a
$$

and

$$
\left\|x_{1}\right\|=\cdots=\left\|x_{n}\right\| .
$$

Proof. From Theorem 1, replacing the vectors $x_{1}, \cdots, x_{n}$, respectively, by the vectors $q_{1} x_{1}, \cdots, q_{n} x_{n}$, one obtains

$$
r\left(q_{1}\left\|x_{1}\right\|+\cdots+q_{n}\left\|x_{n}\right\|\right) \leqq\left\|q_{1} x_{1}+\cdots+q_{n} x_{n}\right\| .
$$

Equality holds if and only if

$$
q_{1} x_{1}+\cdots+q_{n} x_{n}=r\left(q_{1}\left\|x_{1}\right\|+\cdots+q_{n}\left\|x_{n}\right\|\right) a .
$$

Now, from Hardy, Littlewood, and Pólya [4, p. 26], making use here of $q_{1}+\cdots+q_{n}=1$,

$$
\left.\begin{array}{c}
\left\|x_{1}\right\| q_{1} \cdots\left\|x_{n}\right\| q_{n} \\
\left(q_{1}\left\|x_{1}\right\| p+\cdots+q_{n}\left\|x_{n}\right\|{ }^{p}\right)^{1 / p}
\end{array}\right\} \leqq q_{1}\left\|x_{1}\right\|+\cdots+q_{n}\left\|x_{n}\right\|,
$$

with equality if and only if $\left\|x_{1}\right\|=\cdots=\left\|x_{n}\right\|$. This gives the desired result.

Taking $n=2$ in Theorem 1 , with $x_{1}=x$ and $x_{2}=y$, gives $r(\|x\|+\|y\|)$ $\leqq\|x+y\|$; which, upon squaring both sides, yields

$$
r^{2}\|x\| \cdot\|y\|-\frac{1}{2}\left(1-r^{2}\right)\left(\|x\|^{2}+\|y\|^{2}\right) \leqq \operatorname{Re}(x, y),
$$

where equality holds if and only if $x+y=r(\|x\|+\|y\|) a$. This inequality may be regarded as an inequality complementary to Schwarz's inequality. The referee has pointed out that this "is a weakened form of the inequality $\cos 2 \psi=2 r^{2}-1 \leqq \operatorname{Re}(x, y) /\|x\| \cdot\|y\|$, 
which is intuitively clear." This last inequality, upon multiplying through by $2\|x\| \cdot\|y\|$ and then completing the square, becomes

$$
r^{2}(\|x\|+\|y\|)^{2}+\left(1-r^{2}\right)(\|x\|-\|y\|)^{2} \leqq\|x+y\|^{2} .
$$

The referee's inequality may be proved by applying the Schwarz inequality, in the form $-[x, y] \leqq\{[x, x] \cdot[y, y]\}^{1 / 2}$, to the "new" (semi-definite) scalar product $[x, y]=\operatorname{Re}(x, y)-\operatorname{Re}(x, a) \cdot \operatorname{Re}(y, a)$. Upon transposing, and dividing through by $\|x\| \cdot\|y\|$, one obtains

$$
\begin{aligned}
\frac{\operatorname{Re}(x, a)}{\|x\|} \cdot \frac{\operatorname{Re}(y, a)}{\|y\|}-\{[1 & \left.\left.-\left(\frac{\operatorname{Re}(x, a)}{\|x\|}\right)^{2}\right]\left[1-\left(\frac{\operatorname{Re}(y, a)}{\|y\|}\right)^{2}\right]\right\}^{1 / 2} \\
& \leqq \frac{\operatorname{Re}(x, y)}{\|x\| \cdot\|y\|},
\end{aligned}
$$

which implies that $2 r^{2}-1 \leqq \operatorname{Re}(x, y) /\|x\| \cdot\|y\|$.

TheOREM 2. Let $a_{1}, \cdots, a_{m}$ be orthonormal vectors in $H$. Suppose the vectors $x_{1}, \cdots, x_{n}$, whenever $x_{i} \neq 0$, satisfy

$$
0 \leqq r_{k} \leqq \frac{\operatorname{Re}\left(x_{i}, a_{k}\right)}{\left\|x_{i}\right\|} ; \quad i=1, \cdots, n ; k=1, \cdots, m .
$$

Then

$$
\left(r_{1}^{2}+\cdots+r_{m}^{2}\right)^{1 / 2}\left(\left\|x_{1}\right\|+\cdots+\left\|x_{n}\right\|\right) \leqq\left\|x_{1}+\cdots+x_{n}\right\|,
$$

where equality holds if and only if

(16) $x_{1}+\cdots+x_{n}=\left(\left\|x_{1}\right\|+\cdots+\left\|x_{n}\right\|\right)\left(r_{1} a_{1}+\cdots+r_{m} a_{m}\right)$.

Proof. In view of Bessel's inequality, applied to the vector $x_{1}+\cdots+x_{n}$ and the orthonormal sequence $a_{1}, \cdots, a_{m}$,

$$
\begin{aligned}
\left\|x_{1}+\cdots+x_{n}\right\|^{2} & \geqq \sum_{k=1}^{m}\left|\left(x_{1}+\cdots+x_{n}, a_{k}\right)\right|^{2} \\
& \geqq \sum_{k=1}^{m}\left[\operatorname{Re}\left(x_{1}+\cdots+x_{n}, a_{k}\right)\right]^{2} \\
& =\sum_{k=1}^{m}\left[\operatorname{Re}\left(x_{1}, a_{k}\right)+\cdots+\operatorname{Re}\left(x_{n}, a_{k}\right)\right]^{2} .
\end{aligned}
$$

Now, by hypothesis (14),

$$
\operatorname{Re}\left(x_{1}, a_{k}\right)+\cdots+\operatorname{Re}\left(x_{n}, a_{k}\right) \geqq r_{k}\left(\left\|x_{1}\right\|+\cdots+\left\|x_{n}\right\|\right),
$$

which yields (15). 
Now for the equality condition in (15). If (16) holds, then it is clear that equality holds in (15). Suppose the equality sign holds in (15). Then it holds in every intermediate inequality in the argument just given. That is to say, one has
(a) $x_{1}+\cdots+x_{n}=\sum_{k=1}^{m}\left(x_{1}+\cdots+x_{n}, a_{k}\right) a_{k}$,
(b) $\operatorname{Im}\left(x_{1}+\cdots+x_{n}, a_{k}\right)=0, \quad k=1, \cdots, m$,

and

(c) $\operatorname{Re}\left(x_{i}, a_{k}\right)=r_{k}\left\|x_{i}\right\| ; \quad i=1, \cdots, n ; k=1, \cdots, m$.

Hence,

$$
\begin{aligned}
\left(x_{1}+\cdots+x_{n}, a_{k}\right) & =\operatorname{Re}\left(x_{1}+\cdots+x_{n}, a_{k}\right) \\
& =\operatorname{Re}\left(x_{1}, a_{k}\right)+\cdots+\operatorname{Re}\left(x_{n}, a_{k}\right) \\
& =r_{k}\left(\left\|x_{1}\right\|+\cdots+\left\|x_{n}\right\|\right),
\end{aligned}
$$

which, together with (a), gives (16).

REMARK 3. Theorem 2 continues to hold if $m=\infty$, that is, if there are infinitely many vectors $a_{k}$.

REMARK 4. The analogues of Corollaries 1 and 2 follow readily (with $r$ replaced by $\left(r_{1}^{2}+\cdots+r_{m}^{2}\right)^{1 / 2}$ ), and will not be stated separately.

3. Complementary triangle inequality in Banach space. Let $B$ be a Banach space, with real or complex scalars. Then one has the following analogue of Theorem 1 of $\$ 2$.

Theorem 3. Let $F$ be a linear functional of unit norm on B. Suppose the vectors $x_{1}, \cdots, x_{n}$, whenever $x_{i} \neq 0$, satisfy

$$
0 \leqq r \leqq \frac{\operatorname{Re} F x_{i}}{\left\|x_{i}\right\|}, \quad i=1, \cdots, n
$$

Then

$$
r\left(\left\|x_{1}\right\|+\cdots+\left\|x_{n}\right\|\right) \leqq\left\|x_{1}+\cdots+x_{n}\right\|,
$$

where equality holds if and only if both

$$
F\left(x_{1}+\cdots+x_{n}\right)=r\left(\left\|x_{1}\right\|+\cdots+\left\|x_{n}\right\|\right)
$$

and

$$
F\left(x_{1}+\cdots+x_{n}\right)=\left\|x_{1}+\cdots+x_{n}\right\| .
$$


Proof. Since the norm of $F$ is unity, one has $|F x| \leqq\|x\|$ for any $x$ in $B$. Applied to the vector $x_{1}+\cdots+x_{n}$ this inequality yields

$$
\begin{aligned}
\left\|x_{1}+\cdots+x_{n}\right\| & \geqq\left|F\left(x_{1}+\cdots+x_{n}\right)\right| \\
& \geqq\left|\operatorname{Re} F\left(x_{1}+\cdots+x_{n}\right)\right| \\
& =\left|\operatorname{Re} F x_{1}+\cdots+\operatorname{Re} F x_{n}\right| .
\end{aligned}
$$

Now, by hypothesis (17),

$$
\begin{aligned}
\left|\operatorname{Re} F x_{1}+\cdots+\operatorname{Re} F x_{n}\right| & =\operatorname{Re} F x_{1}+\cdots+\operatorname{Re} F x_{n} \\
& \geqq r\left(\left\|x_{1}\right\|+\cdots+\left\|x_{n}\right\|\right),
\end{aligned}
$$

which yields (18).

Now for the equality condition in (18). If (19) and (20) hold, then it is clear that equality holds in (18). Next, suppose the equality sign holds in (18). Then it holds in every intermediate inequality in the argument just given. That is to say, one has

$$
\begin{aligned}
& \text { (a) }\left\|x_{1}+\cdots+x_{n}\right\|=\left|F\left(x_{1}+\cdots+x_{n}\right)\right| \text {, } \\
& \text { (b) } \operatorname{Im} F\left(x_{1}+\cdots+x_{n}\right)=0,
\end{aligned}
$$

and

$$
\text { (c) } \operatorname{Re} F x_{i}=r\left\|x_{i}\right\|, \quad \text { for } i=1, \cdots, n \text {. }
$$

Hence,

$$
\begin{aligned}
F\left(x_{1}+\cdots+x_{n}\right) & =\operatorname{Re} F\left(x_{1}+\cdots+x_{n}\right) \\
& =\operatorname{Re} F x_{1}+\cdots+\operatorname{Re} F x_{n} \\
& =r\left(\left\|x_{1}\right\|+\cdots+\left\|x_{n}\right\|\right),
\end{aligned}
$$

which is (19); and this, together with (a), gives (20).

REMARK 5. As in the case of Theorem 2, analogues of Corollaries 1 and 2 follow readily.

The next theorem bears the same relation to Theorem 3 as Theorem 2 bears to Theorem 1 .

TheOREM 4. Let $F_{1}, \cdots, F_{m}$ be linear functionals on $B$, each of unit norm. Let

$$
c=\sup _{x \neq 0} \frac{\left|F_{1} x\right|^{2}+\cdots+\left|F_{m} x\right|^{2}}{\|x\|^{2}} ;
$$

it then follows that $1 \leqq c \leqq m$. Suppose the vectors $x_{1}, \cdots, x_{n}$, whenever $x_{i} \neq 0$, satisfy 


$$
0 \leqq r_{k} \leqq \frac{\operatorname{Re} F_{k} x_{i}}{\left\|x_{i}\right\|} ; \quad i=1, \cdots, n ; k=1, \cdots, m .
$$

Then

$$
\left(\frac{r_{1}^{2}+\cdots+r_{m}^{2}}{c}\right)^{1 / 2}\left(\left\|x_{1}\right\|+\cdots+\left\|x_{n}\right\|\right) \leqq\left\|x_{1}+\cdots+x_{n}\right\|,
$$

where equality holds if and only if both

(23) $F_{k}\left(x_{1}+\cdots+x_{n}\right)=r_{k}\left(\left\|x_{1}\right\|+\cdots+\left\|x_{n}\right\|\right), \quad k=1, \cdots, m$,

and

$$
\sum_{k=1}^{m}\left[F_{k}\left(x_{1}+\cdots+x_{n}\right)\right]^{2}=c\left\|x_{1}+\cdots+x_{n}\right\|^{2} .
$$

Proof. From the definition of the number $c$, one has

$$
\begin{aligned}
c\left\|x_{1}+\cdots+x_{n}\right\|^{2} & \geqq \sum_{k=1}^{m}\left|F_{k}\left(x_{1}+\cdots+x_{n}\right)\right|^{2} \\
& \geqq \sum_{k=1}^{m}\left[\operatorname{Re} F_{k}\left(x_{1}+\cdots+x_{n}\right)\right]^{2} \\
& =\sum_{k=1}^{m}\left[\operatorname{Re} F_{k} x_{1}+\cdots+\operatorname{Re} F_{k} x_{n}\right]^{2} .
\end{aligned}
$$

Now, by hypothesis (21),

$$
\operatorname{Re} F_{k} x_{1}+\cdots+\operatorname{Re} F_{k} x_{n} \geqq r_{k}\left(\left\|x_{1}\right\|+\cdots+\left\|x_{n}\right\|\right),
$$

which yields (22).

Now for the equality condition in (22). If (23) and (24) hold, then it is clear that equality holds in (22). Next, suppose the equality sign holds in (22). Then it holds in every intermediate inequality in the argument just given. That is to say, one has
(a) $c\left\|x_{1}+\cdots+x_{n}\right\|^{2}=\sum_{k=1}^{m}\left|F_{k}\left(x_{1}+\cdots+x_{n}\right)\right|^{2}$,
(b) $\operatorname{Im} F_{k}\left(x_{1}+\cdots+x_{n}\right)=0, \quad k=1, \cdots, m$,

and
(c) $\operatorname{Re} F_{k} x_{i}=r_{k}\left\|x_{i}\right\| ;$
$i=1, \cdots, n ; k=1, \cdots, m$.

Hence, 


$$
\begin{aligned}
F_{k}\left(x_{1}+\cdots+x_{n}\right) & =\operatorname{Re} F_{k}\left(x_{1}+\cdots+x_{n}\right) \\
& =\operatorname{Re} F_{k} x_{1}+\cdots+\operatorname{Re} F_{k} x_{n} \\
& =r_{k}\left(\left\|x_{1}\right\|+\cdots+\left\|x_{n}\right\|\right), \quad k=1, \cdots, m,
\end{aligned}
$$

which is (23); and this, together with (a), gives (24).

REMARK 6. As usual, analogues of Corollaries 1 and 2 follow easily.

REMARK 7. Theorem 4 contains Theorem 2 as a special case. One need only take $B$ to be the Hilbert space $H$ and the linear functional $F_{k}$ to be given by

$$
F_{k} x=\left(x, a_{k}\right)
$$

for $x$ in $H$, where the $a_{k}$ are as in Theorem 2. From Bessel's inequality $\left|F_{1} x\right|^{2}+\cdots+\left|F_{m} x\right|^{2}=\left|\left(x, a_{1}\right)\right|^{2}+\cdots+\left|\left(x, a_{m}\right)\right|^{2} \leqq\|x\|^{2}$,

and hence $c \leqq 1$. Since it is already known that $1 \leqq c$, it follows that $c=1$. Even if $B$ is a Hilbert space, but the $a_{k}$ 's are not orthogonal, it may happen that $c>1$ (e.g., take $m=2$ and $a_{1}=a_{2}$ ).

\section{REFERENCES}

1. Herbert S. Wilf, Some applications of the inequality of arithmetic and geometric means to polynomial equations, Proc. Amer. Math. Soc. 14 (1963), 263-265.

2. J. B. Diaz and F. T. Metcalf, Complementary inequalities. I: inequalities complementary to Cauchy's inequality for sums of real numbers, J. Math. Anal. Appl. 9 (1964), 59-74.

3. - Complementary inequalities. II: inequalities complementary to the Buniakowsky-Schwarz inequality for integrals, J. Math. Anal. Appl. 9 (1964), 278-293.

4. G. H. Hardy, J. E. Littlewood, and G. Polya, Inequalities, Cambridge University Press, New York, 1959.

UNIVERSITY OF MARYLAND 